\title{
Treatment of Singularities and Quasi-Static Terms in the EFIE Analysis of Planar Structures
}

\author{
Gonzalo Plaza, Francisco Mesa, Member, IEEE, and Francisco Medina, Senior Member, IEEE
}

\begin{abstract}
This paper reports on some mathematical and numerical details of the application of the electric field integral equation (EFIE) method to the analysis of planar structures printed on multilayered substrates. Closed-form expressions for singular and hypersingular terms of the transverse electric field Green's dyadic (TEFGD) are identified so that they can be explicitly extracted out before solving the EFIE. The problems due to the presence of the hypersingular contribution, usually argued to preclude the application of the EFIE to planar structures, are solved. In addition, a low-frequency expansion of the TEFGD corresponding to a single layer substrate is carried out to recognize nonsingular electrostatic-type contributions that can eventually become very important for computational purposes.
\end{abstract}

Index Terms-Dyadic Green's function, electric field integral equation (EFIE), planar structures.

\section{INTRODUCTION}

$\mathbf{T}$ HE APPLICATION of the mixed-potential integral equation (MPIE) method for solving electromagnetic problems in planar layered structures is often described in the literature as considerably more convenient than the use of the electric field integral equation (EFIE) technique [1]-[3]. Two main reasons are usually adduced. The first reason is that the Green's functions involved in the kernels of the MPIE are scalar functions that can be represented by one-dimensional (1-D) Sommerfeld integrals. The second reason is that the Green's function in the EFIE involves singularities of higher order $\left(R^{-3}\right)$ than those appearing in the MPIE $\left(R^{-1}\right)$. In this way, although a few works follow an EFIE formulation [4], [5], this method is not commonly used and it is generally accepted the manifest superiority of MPIE-based techniques. Nevertheless, the present work will try to show that carrying out an appropriate treatment of the scattered electric field, the EFIE technique can be posed in a form very similar to that found for the MPIE, namely, the kernel of the EFIE can be reduced to 1-D Sommerfeld integrals of scalar functions and only $R^{-1}$-type singularities have to be treated. After doing this, the MPIE and the EFIE methods are found comparable and may be regarded as possible alternative and competent techniques to deal with planar layered structures.

Manuscript received October 3, 2000; revised March 29, 2001. This work was supported by the Comisíon Interministerial de Ciencia y Tecnología, Spain, under Project TIC98-0630.

G. Plaza and F. Mesa are with Grupo de Microondas, Departamento de Física Aplicada I, Facultad de Informática, Universidad de Sevilla, 41012-Sevilla, Spain (e-mail: plaza@us.es; mesa@us.es).

F. Medina is with Grupo de Microondas, Departamento de Electrónica y Electromagnetismo, Facultad de Física, Universidad de Sevilla, 41012-Sevilla, Spain (e-mail: medina@us.es).

Publisher Item Identifier S 0018-926X(02)04955-4.
This work will focus on the application of the EFIE to compute the scattered currents on a perfect conductor printed on a multilayered dielectric substrate, which may also show uniaxial anisotropy with its optical axis normal to the interface. In the development of the EFIE problem for this structure, it will be first shown that a convenient analytic preprocessing of the singularities of the transverse electric field Green's dyadic (TEFGD) is the key point to overcome the apparent disadvantages of the EFIE. The study of the singularities in the TEFGD will also make possible an additional understanding of the physical meaning of the singular-terms contribution to the scattered electric field. In a second step, the study will be completed with the obtaining of the most relevant nonsingular terms in the TEFGD for a particular (although representative) structure consisting of a perfect conductor printed on a grounded anisotropic dielectric layer. Special emphasis will be put on those nonsingular terms that are of electrostatic type, whose contribution may become specially significant.

\section{TRANSVERSE EleCtRIC FIELD GREEN's DyADIC (TEFGD)}

It is usually accepted that the most important drawback of the EFIE technique when dealing with planar printed structures is the high-order singularities exhibited by the kernel of the corresponding integral equation, that is, by the TEFGD. In this way, any attempt of improving the efficiency of the EFIE should focus on giving an appropriate treatment for the singularities of this dyadic. This treatment can be greatly simplified if the TEFGD of the multilayered structure, as that shown in Fig. 1, is expressed in a convenient way. Fig. 1 shows a surface current density supported by a perfectly conducting surface $S$ printed on a grounded dielectric layered substrate. Each layer of the substrate is laterally unbounded and can be anisotropic with its optical axis directed along the $y$ axis, namely its permittivity dyadic $\bar{\varepsilon}_{i}$ is given by

$$
\bar{\varepsilon}_{i}=\varepsilon_{0}\left[\varepsilon_{t, i} \overline{\mathbf{I}}_{t}+\varepsilon_{y, i} \hat{\mathbf{y}} \hat{\mathbf{y}}\right]
$$

where symbol ${ }^{\wedge}$ indicates unit vector, $\overline{\mathbf{I}}_{t}=\hat{\mathbf{x}} \hat{\mathbf{x}}+\hat{\mathbf{z}} \hat{\mathbf{z}}$ is the unit transverse dyadic (subscript $t$ will indicate in the following transverse to $y$ axis), and subscript $i$ refers to the $i$ th layer. The constitutive properties of this type of substrate exhibit transverse (to $y$ axis) homogeneity as well as transverse isotropy.

Let us assume an incident (or imposed) electric field, $\mathbf{E}^{\text {inc, }}$ with an implied $\exp (j \omega t)$ time dependence that will not be explicitly written henceforth, that gives rise to a scattered field $\mathbf{E}^{\text {sct }}$. The EFIE to be solved for computing the scattered surface 


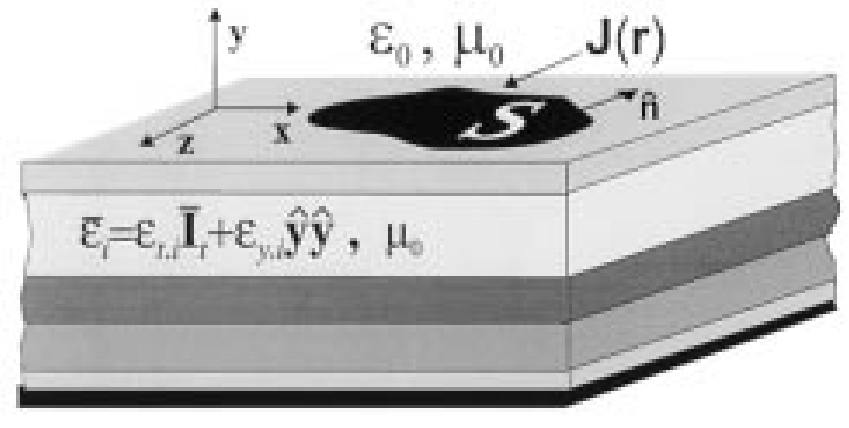

Fig. 1. Planar perfect conductor printed on a uniaxial anisotropic dielectric multilayered substrate with the optical axis along $y$ axis.

current density on the metallized surface, $\mathbf{J}(\mathbf{r})$, can be written as

$$
\hat{\mathbf{y}} \times \mathbf{E}^{\mathrm{tot}}(\mathbf{r})=\hat{\mathbf{y}} \times\left[\mathbf{E}^{\mathrm{inc}}(\mathbf{r})+\mathbf{E}^{\mathrm{sct}}(\mathbf{r})\right]=\mathbf{0} \quad \mathbf{r} \in S
$$

or, equivalently

$$
-\mathbf{E}_{t}^{\text {inc }}(\mathbf{r})=\int_{S} \overline{\mathbf{G}}\left(\mathbf{r}-\mathbf{r}^{\prime}\right) \cdot \mathbf{J}\left(\mathbf{r}^{\prime}\right) d S^{\prime} \quad \mathbf{r} \in S
$$

where $\overline{\mathbf{G}}\left(\mathbf{r}-\mathbf{r}^{\prime}\right)$ is the transverse electric field Green's dyadic and $\mathbf{r}$ and $\mathbf{r}^{\prime}$ are the observation and source points respectively, both on the metallized surface $S$. The explicit dependence on $\left(\mathbf{r}-\mathbf{r}^{\prime}\right)$ exhibited by the Green's dyadic in the previous equation is an immediate consequence of the transverse homogeneity of the dielectric structure. As is well known, the direct computation of TEFGD is rather an impracticable task, which causes $\overline{\mathbf{G}}\left(\mathbf{r}-\mathbf{r}^{\prime}\right)$ to be numerically computed as the inverse transform of its corresponding spectral expression. The obtaining of the spectral expression for the TEFGD of a multilayered and/or anisotropic structure is adequately documented in the open literature, for example [3], [6]-[8]. When computing the inverse Fourier transform of the TEFGD, nonconvergent integrals appear because of the asymptotic linear growing of the TEFGD with the spectral variable. This asymptotic behavior is associated with a hypersingular term (of the type $R^{-3}$ ) appearing in the spatial expression of the TEFGD for $\mathbf{r}=\mathbf{r}^{\prime}$. The existence of this kind of hypersingularity in the electric Green's dyadic, which corresponds to the near field of an elementary dipole, is a general feature of this dyadic that does not depend on the particular dielectric properties of the medium surrounding the source dipole. Since the presence of the hypersingular term in the spatial TEFGD precludes direct numerical computation of the scattered electric field in (3), the practical implementation of the EFIE basically lies on the ability to properly deal with the hypersingular term. It would be then required an adequate extraction of the asymptotic behavior of the spectral expression of the TEFGD as well as the obtaining of a closed form expression of its corresponding singular spatial counterpart.

In order to carry out the aforementioned treatment, the spectral expression of the TEFGD will be first obtained and written in a compact form to simplify the further analysis. Then, closed form expressions for the singular terms in the spatial TEFGD will be obtained by Fourier-Bessel transforming the asymptotic behavior of the spectral expression for the TEFGD. Finally, a simple one-layer anisotropic dielectric grounded substrate will be analyzed in order to obtain the nonsingular electrostatic-type terms in the TEFGD. These terms will be obtained after expanding the TEFGD into a series of powers of the frequency. As it will be explained, they can become as relevant as the singular ones in this practical structure.

\section{A. Spectral TEFGD}

To compute the spectral TEFGD for uniaxial substrates with the optical axis normal to the interface, it is convenient to use polar spectral variables $k_{\rho}$ and $\xi$

$$
\begin{aligned}
k_{\rho} & =\sqrt{k_{x}^{2}+k_{z}^{2}} \\
\xi & =\tan ^{-1}\left(\frac{k_{z}}{k_{x}}\right)
\end{aligned}
$$

where $k_{x}$ and $k_{z}$ are the spectral variables for the Fourier transforms along $x$ and $z$ directions respectively. Thus, the spectral TEFGD $\overline{\mathbf{G}}\left(k_{\rho}, \xi\right)$ can be conveniently written as

$$
\overline{\mathbf{G}}\left(k_{\rho}, \xi\right)=Q\left(k_{\rho}\right) \overline{\mathbf{I}}_{t}+T\left(k_{\rho}\right)\left[2 \hat{\mathbf{k}}_{\rho} \hat{\mathbf{k}}_{\rho}-\overline{\mathbf{I}}_{t}\right]
$$

where $Q\left(k_{\rho}\right)$ and $T\left(k_{\rho}\right)$ are radial spectral functions which, after some algebra, can be computed starting from any of the algorithms above mentioned. (In the following, the spectral/spatial nature of the different quantities will be apparent looking at the corresponding variables.)

A formal expression for the corresponding spatial Greens's dyadic can be now written as the inverse transform of (5), that is

$$
\overline{\mathbf{G}}(\mathbf{R})=S_{0}\left\{\overline{\mathbf{G}}\left(k_{\rho}, \xi\right)\right\}
$$

where $\mathbf{R}=\mathbf{r}-\mathbf{r}^{\prime}$ and

$$
S_{m}\left\{g\left(k_{\rho}\right)\right\}=\frac{1}{2 \pi} \int_{0}^{\infty} k_{\rho} J_{m}\left(k_{\rho} \rho\right) g\left(k_{\rho}\right) d k_{\rho}
$$

stands for the inverse Fourier-Bessel transform of integer order $m$.

Considering now the following identity:

$$
S_{0}\left\{g\left(k_{\rho}\right)\left[2 \hat{\mathbf{k}}_{\rho} \hat{\mathbf{k}}_{\rho}-\overline{\mathbf{I}}_{t}\right]\right\}=S_{2}\left\{g\left(k_{\rho}\right)\right\}\left[\overline{\mathbf{I}}_{t}-2 \hat{\mathbf{R}} \hat{\mathbf{R}}\right]
$$

the spatial TEFGD can be written as

$$
\overline{\mathbf{G}}(\mathbf{R})=S_{0}\left\{Q\left(k_{\rho}\right)\right\} \overline{\mathbf{I}}_{t}+S_{2}\left\{T\left(k_{\rho}\right)\right\}\left[\overline{\mathbf{I}}_{t}-2 \hat{\mathbf{R}} \hat{\mathbf{R}}\right] .
$$

It is interesting to point out that the TEFGD has been expressed in terms of two 1-D Sommerfeld-type integrals of two scalar functions. Similarly, the application of the MPIE formulation also involves two 1-D Sommerfeld-type integrals of two scalar functions. In this way and after a proper treatment of the singularities in the EFIE, the final computational effort becomes quite similar in both schemes.

\section{B. Spatial Expressions for the Singular Terms of the TEFGD}

As is well known, the asymptotic behavior of the spectral TEFGD for $k_{\rho} \gg k_{0}$ ( $k_{0}$ is the free-space wavenumber) is associated with the inverse Fourier-Bessel transform of the electric-field near the dipole source (that is, for $R \ll \lambda_{0}$, where $\lambda_{0}$ is 
the free-space wavelength). Thus, the singular terms in the spatial TEFGD (i.e., the spectral dominant terms in the asymptotic limit) are only related to the layer that the conductor is printed on, namely, the upper one. In this sense, the singular terms in the spatial TEFDG can be obtained from the specific expression of the TEFGD for a single layer structure with the same dielectric properties as the upper layer in the original layered substrate. For this simple case, and assuming that the single layer is grounded (this particular structure will be considered later in Section IV because of its practical interest), the expression for $Q\left(k_{\rho}\right)$ and $T\left(k_{\rho}\right)$ is found to be

$$
\begin{aligned}
& Q\left(k_{\rho}\right)=\frac{j \omega \mu_{0}}{2}\left[\frac{1}{C\left(k_{\rho}\right)}-\frac{1}{\Pi\left(k_{\rho}\right)}\right] \\
& T\left(k_{\rho}\right)=\frac{j \omega \mu_{0}}{2}\left[\frac{1}{C\left(k_{\rho}\right)}+\frac{1}{\Pi\left(k_{\rho}\right)}\right]
\end{aligned}
$$

where

$$
\begin{aligned}
C\left(k_{\rho}\right) & =\frac{k_{0}^{2}}{\sqrt{k_{\rho}^{2}-k_{0}^{2}}}+\frac{\varepsilon_{t} k_{0}^{2} \operatorname{coth}\left(\gamma_{c} h\right)}{\gamma_{c}} \\
\Pi\left(k_{\rho}\right) & =\sqrt{k_{\rho}^{2}-k_{0}^{2}}+\gamma_{\pi} \operatorname{coth}\left(\gamma_{\pi} h\right) \\
\gamma_{c}^{2} & =\frac{\varepsilon_{t}}{\varepsilon_{y}} k_{\rho}^{2}-\varepsilon_{t} k_{0}^{2} \\
\gamma_{\pi}^{2} & =k_{\rho}^{2}-\varepsilon_{t} k_{0}^{2}
\end{aligned}
$$

with $h$ being the layer height. The asymptotic behavior of $\overline{\mathbf{G}}\left(k_{\rho}, \xi\right)$ can be now obtained by expanding functions $Q\left(k_{\rho}\right)$ and $T\left(k_{\rho}\right)$ into a series of powers $k_{\rho}^{1-2 n}$ with $n \geq 0$. This expansion is carried out after assuming that the hyperbolic functions in (12) and (13) have reached its asymptotic values $(\operatorname{coth}(\cdot)=1)$. This assumption has no effect on the singular behavior of the spatial functions for $R \ll \lambda_{0}$ since the hyperbolic functions in (10) and (11) just account for the presence of the ground plane and it is expected that the ground plane will not affect the singular behavior of the field.

After some algebra, the dominant terms in the asymptotic expansion of $Q\left(k_{\rho}\right)$ and $T\left(k_{\rho}\right)$ accounting for the singular spatial behavior of the TEFGD are found to be

$$
\begin{aligned}
Q^{\infty}\left(k_{\rho}\right) & =-\frac{j \omega \mu_{0}(1-\Gamma)}{2 k_{\rho}}-\frac{k_{\rho}}{j \omega \varepsilon_{0} 4 \varepsilon_{\mathrm{eff}}} \\
T^{\infty}\left(k_{\rho}\right) & =\frac{j \omega \mu_{0} \Gamma}{2 k_{\rho}}-\frac{k_{\rho}}{j \omega \varepsilon_{0} 4 \varepsilon_{\mathrm{eff}}}
\end{aligned}
$$

where

$$
\begin{aligned}
\varepsilon_{\mathrm{eff}} & =\frac{1+\sqrt{\varepsilon_{t} \varepsilon_{y}}}{2} \\
\Gamma & =\frac{\sqrt{\varepsilon_{t} \varepsilon_{y}}\left(2-\varepsilon_{y}+\sqrt{\varepsilon_{t} \varepsilon_{y}}\right)}{8 \varepsilon_{\mathrm{eff}}^{2}} .
\end{aligned}
$$

The subscript "eff" in (18) stands for effective, since $\varepsilon_{\text {eff }}$ corresponds to the effective relative permittivity appearing in the computation of the electric field (or potential) on the substrate under electrostatic conditions.
The corresponding spatial expression of the asymptotic radial functions (16) and (17) are now obtained as the following inverse Fourier-Bessel transforms:

$$
\begin{aligned}
& S_{0}\left\{Q^{\infty}\right\}=-\frac{j \omega \mu_{0}(1-\Gamma)}{4 \pi R}+\frac{1}{8 \pi j \omega \varepsilon_{0} \varepsilon_{\mathrm{eff}} R^{3}} \\
& S_{2}\left\{T^{\infty}\right\}=\frac{j \omega \mu_{0} \Gamma}{4 \pi R}-\frac{3}{8 \pi j \omega \varepsilon_{0} \varepsilon_{\mathrm{eff}} R^{3}} .
\end{aligned}
$$

Substituting (20) and (21) into (9) and after straightforward manipulations, the spatial expression for the singular part in the TEFGD can be expressed as

$$
\overline{\mathbf{G}}_{\text {sing }}(\mathbf{R})=\overline{\mathbf{G}}_{(-3)}(\mathbf{R})+\overline{\mathbf{G}}_{(-1)}(\mathbf{R})
$$

where

$$
\begin{aligned}
\overline{\mathbf{G}}_{(-3)}(\mathbf{R}) & =\frac{1}{4 \pi j \omega \varepsilon_{0} \varepsilon_{\mathrm{eff}}}\left[3 \hat{\mathbf{R}} \hat{\mathbf{R}}-\overline{\mathbf{I}}_{t}\right] R^{-3} \\
\overline{\mathbf{G}}_{(-1)}(\mathbf{R}) & =-\frac{j \omega \mu_{0}}{4 \pi}\left[\overline{\mathbf{I}}_{t}-2 \Gamma\left(\overline{\mathbf{I}}_{t}-\hat{\mathbf{R}} \hat{\mathbf{R}}\right)\right] R^{-1} .
\end{aligned}
$$

The complete spatial TEFGD can be then decomposed as follows:

$$
\overline{\mathbf{G}}(\mathbf{R})=\overline{\mathbf{G}}_{(-3)}(\mathbf{R})+\overline{\mathbf{G}}_{(-1)}(\mathbf{R})+\overline{\mathbf{G}}_{\mathrm{reg}}(\mathbf{R})
$$

where $\overline{\mathbf{G}}_{\text {reg }}(\mathbf{R})$ stands for the regular part of the spatial TEFGD. This regular part can be readily computed by numerically reversing to the spatial domain the spectral functions $Q\left(k_{\rho}\right)-$ $Q^{\infty}\left(k_{\rho}\right)$ and $T\left(k_{\rho}\right)-T^{\infty}\left(k_{\rho}\right)$ for a few values of the polar distance $R$ and then properly interpolating to get an accurate approximation for any value of $R$. In practice, the values obtained for $\overline{\mathbf{G}}_{\mathrm{reg}}(\mathbf{R})$ are stored for further analysis of different devices built on the same substrate and operating at the same frequency.

Finally, it is interesting to mention that the expressions for the singular terms in the TEGFD (23) and (24) apply for any multilayered substrate with no restriction about the electric or magnetic properties of its layers except for the upper one, which must be an uniaxial anisotropic dielectric with the optical axis normal to the interface.

\section{SCATtEREd ElEctric Field Due to the SingulaR TERMS IN TEFGD}

Once the exact expression for the singular terms in the TEFGD have been obtained, this section will focus on the computation of the scattered transverse electric field due to these singular terms. First, the complete scattered transverse electric field will be split into two parts: one related to the singular terms in the TEFGD and the other to the regular terms in the TEFGD. Next, and fundamental for further analysis and computation of reaction-type integrals, a simple treatment will show that the contribution to the transverse electric field associated with the $R^{-3}$ singularity in the TEFGD can be conveniently expressed as a convergent integral.

Using the decomposition (25) for the TEFGD, the complete scattered transverse electric field will be written as

$$
\mathbf{E}_{t}(\mathbf{r})=\mathbf{E}_{t(-1)}(\mathbf{r})+\mathbf{E}_{t(-3)}(\mathbf{r})+\mathbf{E}_{t, \operatorname{reg}}(\mathbf{r})
$$


where

$$
\begin{aligned}
& \mathbf{E}_{t(-1)}(\mathbf{r})=-\frac{j \omega \mu_{0}}{4 \pi} \int_{S}\left[\frac{\overline{\mathbf{I}}_{t}-2 \Gamma\left(\overline{\mathbf{I}}_{t}-\hat{\mathbf{R}} \hat{\mathbf{R}}\right)}{R}\right] \cdot \mathbf{J}\left(\mathbf{r}^{\prime}\right) d S^{\prime} \\
& \mathbf{E}_{t(-3)}(\mathbf{r})=\frac{1}{4 \pi \varepsilon_{0} \varepsilon_{\text {eff }}} \int_{S}\left[\frac{3 \hat{\mathbf{R}} \hat{\mathbf{R}}-\overline{\mathbf{I}}_{t}}{R^{3}}\right] \cdot \frac{\mathbf{J}\left(\mathbf{r}^{\prime}\right)}{j \omega} d S^{\prime}
\end{aligned}
$$

and

$$
\mathbf{E}_{t, \mathrm{reg}}(\mathbf{r})=\int_{S} \overline{\mathbf{G}}_{\mathrm{reg}}(\mathbf{R}) \cdot \mathbf{J}\left(\mathbf{r}^{\prime}\right) d S^{\prime}
$$

The indexes $(-1)$ and $(-3)$ do not mean that the corresponding fields are singular with this radial dependence. They simply stand for the term of the TEFGD that they come from. In fact, the electric field created by a surface current density is regular at any point.

Next, the two terms contributing to the electric field related to the singular terms of the TEFGD will be discussed. Both its numerical computation and its physical meaning will be analyzed in detail.

\section{A. $\mathbf{E}_{t(-1)}$ Electric Field}

The integrand in (27) has a $R^{-1}$ singularity for $\mathbf{r}=\mathbf{r}^{\prime}$. However, provided $\mathbf{J}(\mathbf{r})$ is a continuous function [as is expected to occur if $\mathbf{J}(\mathbf{r})$ is a physically valid current density], this improper integral converges. Thus, the practical computation of (27) does not pose any particular numerical problem.

With respect to the physical meaning of this field, it should be noticed that (27) only provides the transverse component of the complete $\mathbf{E}_{(-1)}(\mathbf{r})$ electric field. Nevertheless, only a qualitative description of its nature will be carried out since a complete discussion would require to know the whole expression of $\mathbf{E}_{(-1)}(\mathbf{r})$ in order to obtain the sources of this field. Following a physical rationale, the solenoidal part of $\mathbf{E}_{(-1)}(\mathbf{r})$ can be associated with the time variations of the near magnetic flux density field $\mathbf{B}_{(-2)}(\mathbf{r})$ (a Biot-Savart type field). This magnetic field is due to the surface currents on the conductors and the displacement currents associated with the electrostatic-type electric field $\mathbf{E}_{(-3)}(\mathbf{r})$ [the electrostatic-type nature of $\mathbf{E}_{(-3)}(\mathbf{r})$ will be explicitly shown later]. Analogously, the irrotational part can be related to the surface polarization charge on the substrate interface [note that $\mathbf{E}_{(-1)}(\mathbf{r})=\mathbf{0}$ in Electrostatics, namely, if $\omega=0]$. This surface polarization charge gives rise to the discontinuity of the component of $\mathbf{E}_{(-1)}(\mathbf{r})$ normal to the interface; it is worth to mention that, obviously, this surface polarization charge does not appear if $\mathbf{J}$ is embedded in an homogenous layer.

\section{B. $\mathbf{E}_{t(-3)}$ Electric Field}

The integrand of the contribution (28) to the complete transverse electric field involves a $R^{-3}$ singularity, which, in principle, causes this improper integral not to converge (at least in the classical sense). Thus, expression (28) should be rather considered as a symbolic expression for $\mathbf{E}_{t(-3)}$. When volume current densities are present, the $R^{-3}$ singularity can be treated by using the method of circumvent integrating over the singularity, that is, excluding the volume surrounding the singularity, the so-called exclusion volume. The contribution of this exclusion volume is then accounted for by an appropriate dyadic [9]. Nevertheless, to the authors' knowledge, this technique has not been extended to deal with surface current densities as those appearing in the present analysis. Thus, a different technique to treat the $R^{-3}$ singularity of (28) will be proposed. This technique is based on the formal equivalence of (28) to an electrostatic problem. More specifically, the dyadic in the integrand of (28) can be recognized as that corresponding to the electrostatic field produced by an elementary dipole in an homogeneous medium of relative permittivity $\varepsilon_{\text {eff }}[10]$. Then, expression (28) is formally equivalent to the expression of the electrostatic field due to a surface dipole distribution whose polarization, $\mathbf{P}$, is given by

$$
\mathbf{P}(\mathbf{r})=\frac{\mathbf{J}(\mathbf{r})}{j \omega}
$$

This formal equivalence to an electrostatic problem makes it possible to express (28) as a convergent improper integral. Thus, following the usual procedure in electrostatics, $\mathbf{E}_{t(-3)}$ can be expressed in terms of an equivalent linear charge density $\lambda$ and an equivalent surface charge density $\sigma$ associated with the surface dipole distribution (30), that is

$$
\mathbf{E}_{t(-3)}(\mathbf{r})=-\nabla_{t} \int_{\partial S} \frac{\lambda\left(l^{\prime}\right)}{4 \pi \varepsilon_{\mathrm{eff}} R} d l^{\prime}-\nabla_{t} \int_{S} \frac{\sigma\left(\mathbf{r}^{\prime}\right)}{4 \pi \varepsilon_{\mathrm{eff}} R} d S^{\prime}
$$

where

$$
\begin{aligned}
& \lambda(l)=\mathbf{P}(\mathbf{r}) \cdot \hat{\mathbf{n}}=\frac{\mathbf{J}(\mathbf{r})}{j \omega} \cdot \hat{\mathbf{n}} \\
& \sigma(\mathbf{r})=-\nabla_{t} \cdot \mathbf{P}(\mathbf{r})=-\frac{\nabla_{t} \cdot \mathbf{J}(\mathbf{r})}{j \omega}
\end{aligned}
$$

with $\nabla_{t} \equiv \hat{\mathbf{x}}(\partial / \partial x)+\hat{\mathbf{z}}(\partial / \partial z)$ being the transverse nabla operator and $\hat{\mathbf{n}}$ the normal vector pointing outward to $\partial S$ (see Fig. 1). Provided that the scattered surface currents (or, equivalently, the proposed basis functions for the surface currents when a Method of Moments is employed to solve the problem [11]) have continuous normal components, no linear charge distribution will be present in the problem, i.e., $\lambda(l)=0$. In consequence, the electric field accounting for the $R^{-3}$-type singularity can be finally written as

$$
\mathbf{E}_{t(-3)}(\mathbf{r})=-\nabla_{t} \phi_{(-3)}(\mathbf{r})
$$

namely, $\mathbf{E}_{t(-3)}$ is a purely irrotational field that can be obtained as the transverse gradient of a scalar potential, $\phi_{(-3)}$, given by

$$
\phi_{(-3)}(\mathbf{r})=\int_{S} \frac{\sigma\left(\mathrm{r}^{\prime}\right)}{4 \pi \varepsilon_{\mathrm{eff}} R} d S^{\prime}
$$

After the application of the transformation in the previous equation, the contribution to the complete transverse electric field $\mathbf{E}_{t}$ coming from the singular terms in the TEFGD have been expressed as integrals that only involve $R^{-1}$-type singularities. In this sense, the treatment reported here for the singularities of the TEFGD has overcome the major disadvantage usually 
related to the use of the EFIE method. Thus, the EFIE shows a well-conditioned numerical behavior very similar to that found when using an MPIE-based technique. It is interesting to point out that the potential technique has been only applied to account for the hypersingular contribution to the electric field, whereas the remaining parts are expressed in terms of the TEFGD, that is

$$
\begin{aligned}
\mathbf{E}_{t}(\mathbf{r})=-\nabla_{t} \phi_{(-3)}+\int_{S} \overline{\mathbf{G}}_{-1}(\mathbf{R}) \cdot \mathbf{J}\left(\mathbf{r}^{\prime}\right) d S^{\prime} \\
\quad+\int_{S} \overline{\mathbf{G}}_{\mathrm{reg}}(\mathbf{R}) \cdot \mathbf{J}\left(\mathbf{r}^{\prime}\right) d S^{\prime} .
\end{aligned}
$$

In light of this equation, the present analysis could be interpreted as a hybrid EFIE/MPIE formulation.

Finally, it should be mentioned that a similar treatment of the $R^{-3}$-type singularity was reported by Bressan and Conciauro in [12] to deal with volume currents in a homogeneous isotropic medium. In such homogeneous cases, $\mathbf{E}_{(-3)}$ accounts for the complete contribution to the irrotational part of the electric field due to the singular terms in the Green's dyadic. However, in non homogeneous structures as those considered in this work, the already mentioned polarization surface charge density at the dielectric interface for $\omega \neq 0$ [i.e., the scalar sources of $\mathbf{E}_{(-1)}$ ] appears as an additional source of the complete irrotational part of the electric field due to the singular terms in TEFGD.

\section{Electrostatic-Type Nonsingular TeRms In TEFGD}

Although the contribution to the electric field due to the singular terms in the TEFGD is the most relevant one at short distances to the source, there are many practical structures in which other terms can be similarly meaningful. This situation is found, for example, in a single layer grounded substrate whose height $h$ is significantly smaller than the free-space wavelength $\lambda_{0}$. In such structure it is found that the contribution to the regular transverse electric field $\mathbf{E}_{t, \text { reg }}$ coming from the nonsingular electrostatic-type terms involved in the TEFGD can be as relevant as the $\mathbf{E}_{t(-1)}$ field. This section will focus on the study of the aforementioned nonsingular electrostatic-type terms for this particular and practical structure. As in previous sections, the layer is assumed to be dielectric either isotropic or uniaxial anisotropic with its optical axis directed normal to the interface.

Assuming that these nonsingular electrostatic-type terms in the TEFGD come from the infinite electrostatic images of an elementary dipole placed at the dielectric/air interface, the subsequent image terms will show a dependence of the type $\left(R^{2}+\right.$ $\left.h_{\text {image }}^{2}\right)^{-3 / 2}$, where $h_{\text {image }}$ is the corresponding vertical distance of the considered image with respect to the interface (the specific values of $h_{\text {image }}$ will be found later). Due to the fast decay of these terms as $R$ increases, it is reasonable to expect their contribution to $\mathbf{E}_{t, \text { reg }}$ to be significant only if the typical linear dimension $d$ of the metallized surface is small compared with $\lambda_{0}$ (the remaining nonsingular nonelectrostatic terms are only expected to be more relevant than the electrostatic terms at distances $>\lambda_{0}$ ).

In the forthcoming analysis, the most relevant nonsingular terms in TEFGD will be obtained by expanding the TEFGD in terms of a series of the operating frequency $\omega$. This expansion will also give the singular terms already obtained in a simpler way in Section II-B. The frequency expansion would then provide an alternative approach to obtain the singular and other representative terms in the TEFGD. The obtaining of all these terms and their corresponding analytical preprocessing can be very useful for the efficient computation of the reaction integrals involving $\mathbf{E}_{t, \text { reg. }}$.

Looking at expressions (10) and (11) for the radial spectral functions, it can be observed that both $Q\left(k_{\rho}, \omega\right)$ and $T\left(k_{\rho}, \omega\right)$ are odd functions of the variable $\omega$. Therefore, they can be expanded into series of odd powers of $\omega$ as follows:

$$
\begin{aligned}
& Q\left(k_{\rho} ; \omega\right)=\sum_{m=-1}^{\infty} q_{2 m+1}\left(k_{\rho}\right) \omega^{2 m+1} \\
& T\left(k_{\rho} ; \omega\right)=\sum_{m=-1}^{\infty} t_{2 m+1}\left(k_{\rho}\right) \omega^{2 m+1}
\end{aligned}
$$

where the dominant terms are found to be

$$
\begin{aligned}
q_{-1}\left(k_{\rho}\right)= & t_{-1}\left(k_{\rho}\right)=\frac{-k_{\rho}}{j 2 \varepsilon_{0}\left[1+\varepsilon_{\mathrm{eq}} \operatorname{coth}\left(k_{\rho} h_{\mathrm{eq}}\right)\right]} \\
q_{1}\left(k_{\rho}\right)= & -j \mu_{0}\left[\frac{1}{2 k_{\rho}}-\frac{e^{-2 h k_{\rho}}}{2 k_{\rho}}\right]+t_{1}\left(k_{\rho}\right) \\
t_{1}\left(k_{\rho}\right)= & \frac{j \mu_{0}}{2 k_{\rho}\left[1+\operatorname{coth}\left(k_{\rho} h\right)\right]}-\frac{j \mu_{0}}{4\left[1+\varepsilon_{\mathrm{eq}} \operatorname{coth}\left(k_{\rho} h_{\mathrm{eq}}\right)\right]^{2}} \\
& \times\left\{\frac{\varepsilon_{y} \varepsilon_{\mathrm{eq}} \operatorname{coth}\left(k_{\rho} h_{\mathrm{eq}}\right)}{k_{\rho}}+\varepsilon_{y} \varepsilon_{\mathrm{eq}} h_{\mathrm{eq}}\right. \\
& \left.\times \operatorname{csch}^{2}\left(k_{\rho} h_{\mathrm{eq}}\right)+\frac{1}{k_{\rho}}\right\}
\end{aligned}
$$

with

$$
\begin{aligned}
& \varepsilon_{\mathrm{eq}}=\sqrt{\varepsilon_{y} \varepsilon_{t}} \\
& h_{\mathrm{eq}}=h \sqrt{\frac{\varepsilon_{t}}{\varepsilon_{y}}}
\end{aligned}
$$

(subscript "eq" stands for equivalent). The introduction of the equivalent permittivity and height formally reduces the computation of either $q_{-1}\left(k_{\rho}\right)$ or $t_{-1}\left(k_{\rho}\right)$ for the possible anisotropic uniaxial problem to a simpler isotropic problem [note that for an isotropic substrate of relative permittivity $\varepsilon$, it is found from (40) and (41) that $\varepsilon_{\mathrm{eq}}=\varepsilon$ and $h_{\mathrm{eq}}=h$ ].

As is apparent in light of expansions (35) and (36), the $q_{-1}\left(k_{\rho}\right) \omega^{-1}$ and $t_{-1}\left(k_{\rho}\right) \omega^{-1}$ terms account for the electrostatic-type contribution, since the remaining terms vanish as $\omega \rightarrow 0$. Then, the electrostatic-type part in the TEFGD, $\overline{\mathbf{G}}_{\text {elst }}$, can be readily obtained by taking $Q\left(k_{\rho}\right)=T\left(k_{\rho}\right)=q_{-1}\left(k_{\rho}\right) \omega^{-1}$ in (9), that is

$$
\begin{aligned}
& \overline{\mathbf{G}}_{\text {elst }}(\mathbf{R}) \\
& \quad=\frac{1}{\omega}\left[S_{0}\left\{q_{-1}\left(k_{\rho}\right)\right\} \overline{\mathbf{I}}_{t}+S_{2}\left\{q_{-1}\left(k_{\rho}\right)\right\}\left(\overline{\mathbf{I}}_{t}-2 \hat{\mathbf{R}} \hat{\mathbf{R}}\right)\right] .
\end{aligned}
$$


In order to perform the inverse Fourier-Bessel transformation in (42), it is convenient to write previously $q_{-1}\left(k_{\rho}\right)$ as the following series of exponential functions of $k_{\rho}$ :

$$
\begin{aligned}
q_{-1}\left(k_{\rho}\right) & =\frac{-k_{\rho}}{4 j \varepsilon_{0} \varepsilon_{\mathrm{eff}}}\left\{1+\frac{2 \varepsilon_{\mathrm{eq}}}{1+\varepsilon_{\mathrm{eq}}}\right. \\
& \left.\times \sum_{n=1}^{\infty}(-1)^{n}\left(\frac{\varepsilon_{\mathrm{eq}}-1}{\varepsilon_{\mathrm{eq}}+1}\right)^{n-1} \exp \left(-k_{\rho} H_{n}\right)\right\}
\end{aligned}
$$

where $H_{n}=2 n h_{\text {eq }}$. Now substituting (43) into (42) and after taking the term by term inverse Fourier-Bessel transformation, $\overline{\mathbf{G}}_{\text {elst }}(\mathbf{R})$ can be finally written as

$$
\overline{\mathbf{G}}_{\text {elst }}(\mathbf{R})=\overline{\mathbf{G}}_{\text {elst }}^{(0)}(\mathbf{R})+\sum_{n=1}^{\infty} \overline{\mathbf{G}}_{\text {elst }}^{(n)}(\mathbf{R})
$$

where

$$
\begin{aligned}
\overline{\mathbf{G}}_{\text {elst }}^{(0)}(\mathbf{R})= & \frac{1}{4 \pi j \omega \varepsilon_{0} \varepsilon_{\mathrm{eff}}}\left[\frac{3 \hat{\mathbf{R}} \hat{\mathbf{R}}-\overline{\mathbf{I}}_{t}}{R^{3}}\right] \\
\overline{\mathbf{G}}_{\text {elst }}^{(n)}(\mathbf{R})= & \frac{(-1)^{n}}{4 \pi j \omega \varepsilon_{0} \varepsilon_{\mathrm{eff}}} \cdot\left(\frac{\varepsilon_{\mathrm{eq}}-1}{\varepsilon_{\mathrm{eq}}+1}\right)^{n-1} \frac{2 \varepsilon_{\mathrm{eq}}}{\left(\varepsilon_{\mathrm{eq}}+1\right)} \\
& \times\left[\frac{3 R^{2}\left(\hat{\mathbf{R}} \hat{\mathbf{R}}-\overline{\mathbf{I}}_{t}\right)}{\left(R^{2}+H_{n}^{2}\right)^{5 / 2}}\right] .
\end{aligned}
$$

The first zero-order term in (45) is recognized as the $R^{-3}$ singular term previously found in (23), that is, $\overline{\mathbf{G}}_{\text {elst }}^{(0)}(\mathbf{R}) \equiv \overline{\mathbf{G}}_{(-3)}(\mathbf{R})$. Therefore, as it was explained in Section III, this singular term can be seen as the Green's dyadic associated with the transverse electrostatic field due to an elementary dipole in an homogeneous isotropic medium whose polarization is $\mathcal{P}^{(0)}(\mathbf{r})=(\hat{\mathbf{x}}+\hat{\mathbf{z}}) \delta(\mathbf{r}) / j \omega$. The remaining nonsingular electrostatic-type terms, (46), can be seen as the Green's dyadics related to the transverse electric field due to the infinite electrostatic image dipole distributions $\boldsymbol{P}^{(n)}(\mathrm{r})$ of the source dipole distribution $\boldsymbol{P}^{(0)}(\mathbf{r})$

$$
\begin{aligned}
\boldsymbol{P}^{(n)}(\mathbf{r})=(-1)^{n}\left(\frac{\varepsilon_{\mathrm{eq}}-1}{\varepsilon_{\mathrm{eq}}+1}\right)^{n-1} \frac{2 \varepsilon_{\mathrm{eq}}}{\left(\varepsilon_{\mathrm{eq}}+1\right)} \frac{(\hat{\mathbf{x}}+\hat{\mathbf{z}})}{j \omega} \\
\times \delta\left(\mathbf{r}+H_{n} \hat{\mathbf{y}}\right) .
\end{aligned}
$$

Unlike the hypersingular $\overline{\mathbf{G}}_{\text {elst }}^{(0)}(\mathbf{R})$ dyadic, the dyadic terms in (46) are regular. Thus, their contribution to the transverse electric field, which can be expressed as

$$
\mathbf{E}_{\text {elst }}(\mathbf{r})=\sum_{n=1}^{\infty} \mathbf{E}_{\text {elst }}^{(n)}(\mathbf{r})=\sum_{n=1}^{\infty} \int_{S} \overline{\mathbf{G}}_{\mathrm{elst}}^{(n)}(\mathbf{R}) \cdot \mathbf{J}\left(\mathbf{r}^{\prime}\right) d S^{\prime}
$$

can be directly obtained by numerically computing the integrals appearing in (46).

Taking into account the term-by-term relationship between expressions (46) and (47), it is possible to find an alternative way of expressing $\mathbf{E}_{\text {elst }}(\mathbf{r})$ in terms of electrostatic-type potentials due to the image series of the equivalent surface dipole distribution (30), as it was made in (32) for the hypersingular case. Thus, each term of $\mathbf{E}_{\text {elst }}(\mathbf{r})$ can be written as

$$
\mathbf{E}_{\text {elst }}^{(n)}(\mathbf{r})=\int_{S} \overline{\mathbf{G}}_{\mathrm{elst}}^{(n)}(\mathbf{R}) \cdot \mathbf{J}\left(\mathrm{r}^{\prime}\right) d S^{\prime}=-\nabla_{t} \phi^{(n)}(\mathbf{r})
$$

that is, as the transverse gradient of the following scalar potential:

$$
\phi^{(n)}(\mathrm{r})=\int_{S} \frac{\sigma^{(n)}\left(\mathrm{r}^{\prime}\right)}{4 \pi \varepsilon_{0} \varepsilon_{\mathrm{eff}} \sqrt{R^{2}+H_{n}^{2}}} d S^{\prime}
$$

where

$$
\sigma^{(n)}(\mathbf{r})=-\nabla_{t} \cdot \mathbf{P}^{(n)}(\mathbf{r})
$$

and

$$
\mathbf{P}^{(n)}(\mathbf{r})=(-1)^{n}\left(\frac{\varepsilon_{\mathrm{eq}}-1}{\varepsilon_{\mathrm{eq}}+1}\right)^{n-1} \frac{2 \varepsilon_{\mathrm{eq}}}{\left(\varepsilon_{\mathrm{eq}}+1\right)} \frac{\mathbf{J}(\mathrm{r})}{j \omega} .
$$

The previous expansion of $\mathbf{E}_{\mathrm{elst}}(\mathbf{r})$ in terms of the transverse gradient of certain scalar potentials could be very useful when computing the reaction integrals appearing in the application of the method of moments (MoM). Following the usual treatment reported in the literature [12], [11], the use of the reported decomposition together with its analytical preprocessing would allow to obtain possible closed form expressions for the corresponding reaction integrals.

Finally, it will be briefly considered the nonelectrostatic dominant terms at low frequencies in expansions (35) and (36), namely $q_{1}\left(k_{\rho}\right)$ and $t_{1}\left(k_{\rho}\right)$. Expanding the hyperbolic functions in (38) and (39) into exponential terms, it can be written that

$$
\begin{aligned}
q_{1}\left(k_{\rho}\right) \omega= & -j \omega \mu_{0} \frac{1}{2 k_{\rho}}+j \omega \mu_{0} \frac{1}{4 k_{\rho}} \frac{\varepsilon_{\mathrm{eq}}\left(2-\varepsilon_{y}+\varepsilon_{\mathrm{eq}}\right)}{\left(1+\varepsilon_{\mathrm{eq}}\right)^{2}} \\
& + \text { exponential terms involving } h \\
t_{1}\left(k_{\rho}\right) \omega= & j \omega \mu_{0} \frac{1}{4 k_{\rho}} \frac{\varepsilon_{\mathrm{eq}}\left(2-\varepsilon_{y}+\varepsilon_{\mathrm{eq}}\right)}{\left(1+\varepsilon_{\mathrm{eq}}\right)^{2}} \\
& + \text { exponential terms involving } h .
\end{aligned}
$$

Now recognizing the $h$-independent terms in the previous expressions as the $k_{\rho}^{-1}$-terms in expressions (16) and (17), the spatial expression for $\overline{\mathbf{G}}_{(-1)}(\mathbf{R})$ in (24) can be again obtained after performing inverse transforms. It is interesting to note that the results obtained in the present section related to the singular terms in the TEFGD plainly justify the approximation assumed in Section II-B [i.e., $\operatorname{coth}(*)=1$ ] to compute these terms. Concerning the remaining nonsingular $h$-dependent terms in (53) and (54), it should be mentioned that, to the authors' knowledge, their spatial counterpart cannot be expressed in closed form by analytically performing the corresponding Fourier-Bessel transform. Thus, considering that in most cases these terms are less relevant than the singular terms or than the nonsingular electrostatic ones, they have not been extracted out explicitly. 


\section{CONCLUSION}

Some important details concerning the application of the EFIE to the computation of the current density scattered by printed perfect conductors on iso/anisotropic uniaxial dielectric multilayered substrates have been examined. The singular contributions to the transverse electric field Green's dyadic have been identified and extracted out from the complete dyadic. It has been shown that the major drawback of the application of EFIE to the aforementioned type of problems, namely, the existence of a $R^{-3}$ hypersingularity in the TEFGD, can be overcome by means of a suitable analytical treatment. This treatment is based on Fourier-Bessel inverse transformation of the asymptotic behavior of the spectral Green's function. By exploiting a certain formal equivalence with an electrostatic-type problem, the original EFIE is modified in such a way that the new integral equation only involves a kernel with $R^{-1}$-type singularities. Although singular terms are dominant at very short distances to the source point, non singular electrostatic-type terms in the TEFGD can become significant under certain conditions (namely, when $h \ll \lambda_{0}$, with $h$ being the height of the upper layer). For this reason, these terms have been also recognized in the spatial domain and extracted out for a simple, although practical, one layer structure.

\section{REFERENCES}

[1] D. C. Chang and J. X. Zheng, "Electromagnetic modeling of passive circuits elements in MMIC," IEEE Trans. Microwave Theory Tech., vol. 40, pp. 1741-1747, Sept. 1992.

[2] J. Sercu, N. Faché, F. Libbrecht, and P. Lagase, "Mixed potential integral equation technique for hybrid microstrip-slotine multilayered circuits using a mixed rectangular-triangular mesh," IEEE Trans. Microwave Theory Tech., vol. 43, pp. 1162-1172, May 1995.

[3] K. A. Michalski and J. R. Mosig, "Multilayered media Green's functions in integral equations formulations," IEEE Trans. Antennas Propagat., vol. 45, pp. 508-519, Mar. 1997.

[4] J. Sercu, N. Faché, F. Libbrecht, and D. De Zutter, "Full-Wave spacedomain analysis of open microstrip discontinuities including the singular current-edge behavior," IEEE Trans. Microwave Theory Tech., vol. 41, pp. 1581-1588, Sept. 1993.

[5] A. Toscano and L. Vegni, "Spectral dyadic Green's function formulation for planar integrated structures with a grounded chiral slab," J. Electromagn. Waves Applicat., vol. 6, no. 5-6, pp. 751-769, 1992.

[6] C. M. Krowne, "Fourier transform matrix method of finding propation characteristics of complex anisotropic layered media," IEEE Trans. Microwave Theory Tech., vol. MTT-32, pp. 1617-1625, Dec. 1984.

[7] R. Marqués and M. Horno, "On the spectral dyadic Green's function for stratified linear media," Proc. Inst. Elect. Eng. H, vol. 134, June 1987.

[8] F. L. Mesa, R. Marqués, and M. Horno, "A general algorithm for computing the bidimensional spectral Green's dyad in multilayered complex bianisotropic media: the equivalent boundary method," IEEE Trans. Microwave Theory Tech., vol. 39, pp. 1640-1649, Sept. 1991.

[9] J. van Bladel, "Some remarks on Green's dyadic for inifinte space," IEEE Trans. Antennas Propagat., vol. AP-9, pp. 563-566, 1961.
[10] J. D. Jackson, Classical Electrodynamics, 3rd ed. New York: Wiley, 1999.

[11] S. M. Rao, D. R. Wilton, and A. W. Glisson, "Electromagnetic scattering by surfaces of arbitrary shape," IEEE Trans. Antennas Propagat., vol. AP-30, pp. 409-418, May 1982.

[12] M. Bressan and G. Conciauro, "Singularity extraction from the electric Green's function for a spherical resonator," IEEE Trans. Microwave Theory Tech., vol. MTT-33, pp. 407-414, May 1985.

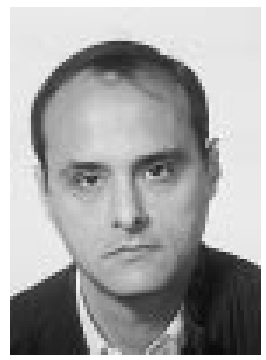

Gonzalo Plaza was born in Cádiz, Spain, on November 26, 1960. He received the Lic. and Dr. degrees in physics from the University of Seville, Seville, Spain, in 1986 and 1995, respectively.

$\mathrm{He}$ is currently an Associate Professor in the Department of Applied Physics I, University of Seville. Presently, his research interest focuses on electromagnetic propagation along planar lines with general materials.

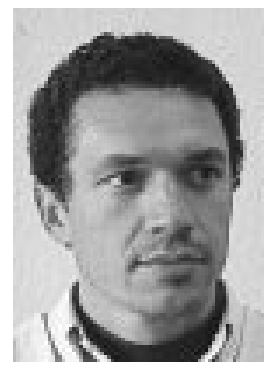

Francisco Mesa (M'94) was born in Cádiz, Spain, on April 15, 1965. He received the Lic. and Dr. degrees from the University of Seville, Seville, Spain, in 1989 and 1991, respectively, both in physics.

$\mathrm{He}$ is currently an Associate Professor in the Department of Applied Physics I, University of Seville. His research interest focuses on electromagnetic propagation/radiation in planar lines with general anisotropic materials.

Francisco Medina (M'90-SM'01) was born in Puerto Real, Cádiz, Spain, in November 1960. He received the Lic. and Dr. degrees from the University of Seville, Seville, Spain, in 1983 and 1987, respectively, both in physics.

From 1986 to 1987, he spent the academic year at the Laboratoire de Microondes de l'ENSEEIHT, Toulouse, France. From 1985 to 1989, he was a Profesor Ayudante with the Department of Electronics and Electromagnetism, University of Seville, and since 1990, he has been Profesor Titular of Electromagnetism. He is currently the Head of the Microwaves Group, University of Seville. His research interests include analytical and numerical methods for planar structures and circuits and the influence on these circuits of anisotropic materials

Dr. Medina was a member of the TPC of the 23rd European Microwave Conference, Madrid, Spain (1993) and a member of the Spanish TPC of the ISRAMT Conference, Málaga, Spain (1999). He is on the editorial board of the IEEE TRANSACTIONS ON MiCROWAVE THEORY AND TECHNIQUES and acts as Reviewer for other IEEE and IEE journals. He was the recipient of a Ministerio de Educación y Ciencia/Ministere de la Recherche et la Technologie Scholarship. 